\title{
Palmprint Identification using Log Transformation of Transform Domain Features
}

\author{
K. P. Shashikala \\ Department of ECE, \\ Dayananda Sagar College of Engineering, \\ Bangalore, India
}

\begin{abstract}
The Palmprint is an efficient physiological biometric trait to identify a person. In this paper we propose Palmprint Identification using Log Transformation of Transform Domain Features. The Region of Interest (ROI) of palmprint image is extracted using preprocessing. The KWT and DWT are applied on preprocessed image to generate features. The KWT and DWT features of test image and database images are compared using Euclidian distance to compute EER and TSR values. The EER and TSR values of KWT and DWT are fused using Log Transformation to get better performance parameters. It is observed that the values of performance parameters are better in the case of proposed algorithm compared to existing algorithms.
\end{abstract}

Keywords- Palmprint, KWT, DWT, ED, EER, TSR.

\section{I.INTRODUCTION}

Biometrics is a technique for measuring the unique human characteristics for identification or verification. Palmprint biometric is rich with substantial and discriminating information consisting of unique low resolution features like principle lines, wrinkles and high resolution texture features such as minutiae points, ridges, bifurcations etc. These features can be fused to obtain a more robust system.

Contribution: In this paper Test and database palmprint features are extracted using KWT and DWT and the TSR and EER performance parameters are computed. The performance parameters are fused at matching level using Log transformation to improve performance of the algorithm.

\section{LITERATURE SURVEY}

Kekre et al., [1] propose a novel Kekre's wavelet Transform which is generated from Kekre's Transform. This is a faster Transform which can be used for various image processing applications. In this paper they have implemented Steganography. Eryun Liu et al., [2] proposed a fine to course matching strategy involving minutiae clustering and minutiae match propagation for Latent palmprint matching. Haifeng Sang et al., [3] use skin color thresholding and hand valley detection for extracting palmprint ROI. Local Binary Pattern (LBP) is applied to obtain features. Chi- Square Statistics used for classification. Jingyuguo et al., [4] proposed feature extraction by Entropy Map and 2DPCA. The effect of

\author{
K. B. Raja \\ Department of ECE, \\ University Visveswaraya College of Engineering, \\ Bangalore University, Bangalore, India
}

illumination and Dimensionality are reduced. Nearest neighbor Classification is used. Khalifa et al., [5] proposed feature extraction by Wavelet, Gabor filter and Co-occurrence matrix and SVM for matching.

\section{DEFINITIONS}

The definitions used for the performance analysis are given below.

A. False Acceptance Rate (FAR) it is the measure of the percentage of falsely accepted palmprints. It is the ratio of the number of invalid palmprints accepted to the total number of palmprints present in outside database as given in equation 1 .

$$
\mathrm{FAR}=\frac{\text { No. of invalid palmprints accepted }}{\text { No. of palmprints in outside the database }}
$$

B. False Rejection Rate (FRR): It is the ratio of number of valid palmprints rejected to the total number of persons in the database as given in equation2. $\mathrm{FRR}=\frac{\text { No. of valid palmprints rejected }}{\text { No. of palmprints in the database }}$

C. Equal Error Rate (EER) It is the rate at which accept and reject rates are equal. It is obtained from the plot of FAR and FRR with respect to threshold as given in equation 3 .

$$
\mathrm{EER}=\mathrm{FAR}=\mathrm{FRR}
$$

D. Total Success Rate (TSR) gives the number of persons correctly matched out of the total number of persons present in the database as given in equation 4 .

$$
\text { TSR }=\frac{\text { No.of Test images Correctly Matched }}{\text { Total no. of persons in Database }}
$$




\section{IV.PROPOSED MODEL}

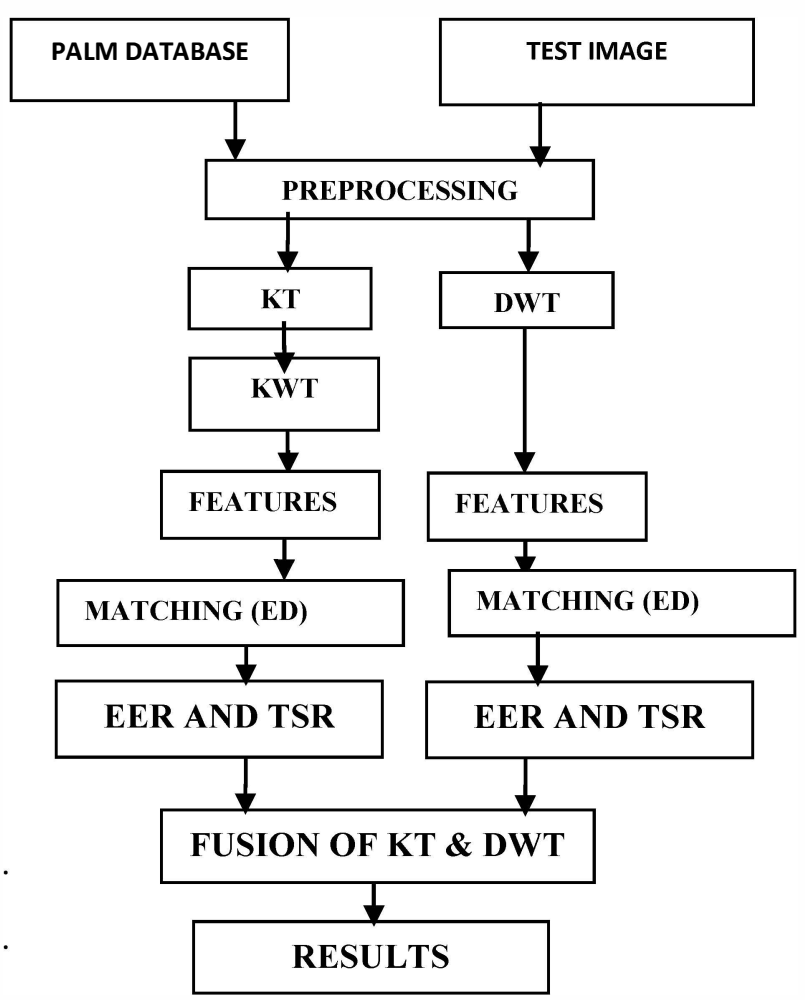

Figure: 1 Proposed Model

The block diagram of the proposed model is as shown in the figure1. The features are extracted using KWT and DWT. The Euclidean Distance is used to compare features of test images with features of images in the database. The matching scores are fused at matching level. The performance parameter values are improved since performance parameters scores using KWT and DWT are fused at matching level using Log transformation.

\section{A . DataBase:}

The Poly U database consists of palmprint images of 386 persons with 20 images per person. Every eighteenth image in the database is considered as test image. The different combinations of number of persons inside and outside database is considered in the range of $20 ; 100,40: 80,60: 60,80: 40$ and 100:20 to compute FRR, TSR, FAR and EER for both the KWT and DWT respectively.

\section{B. Preprocessing}

The original palmprint image of size $384 \times 284$ is converted into binary image to obtain proper edges. The Canny edge detection is applied on the binary image to get boundary of the palmprint. The ROI is obtained using linear equations and normalization. The image is cropped to 170X170. The Histogram Equalization is applied on the ROI for contrast enhancement. The image is again resized to $64 \mathrm{X} 64$.The original image, Binary image, Edge detected image, ROI Detection Scheme, Normalized palmprint, ROI and Histogram Equalized ROI are shown in Figures 2.



(a) Original image

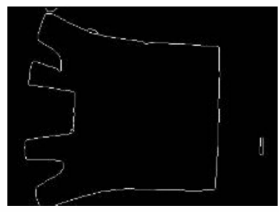

(c)Edge detected image

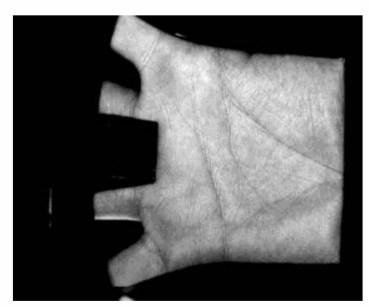

(e)Normalized image

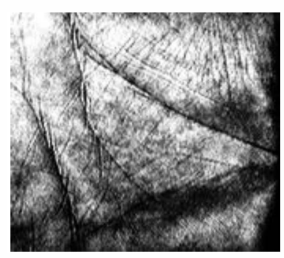

(g) Histogram Equalized ROI

Figure 2: Preprocessing of Palmprint.

The valley points $v 1(k 1, k 2)$ and $v 2(k 3, k 4)$ are located by comparing the distances between the midpoint of the image and the borders of the palm as shown in Figure 3.The midpoint $m(m 1, m 2)$ of the line joining $v 1$ and $v 2$ is found using Equation (5) and (6).

$\mathrm{m} 1=\frac{(\mathrm{k} 1+\mathrm{k} 3)}{2} \ldots \ldots .(5)$

$\mathrm{m} 2=\frac{(\mathrm{k} 2+\mathrm{k} 4)}{2}$

Slope of the perpendicular bisector of $v 1 v 2$ is then found out using Equation (7).

$\mathrm{S}=\frac{-(\mathrm{k} 3-\mathrm{k} 1)}{(\mathrm{k} 4-\mathrm{k} 2)}$

The point's $\mathrm{p}\left(x_{2}, y 2\right)$ and $q(x 3, y 3)$ are obtained using the Equation (8)

. $(\mathrm{x} 2-\mathrm{m} 1) * \mathrm{~s}=(\mathrm{y} 2-\mathrm{m} 2)$

The points $p$ and $q$ lie on the same line but at different distances. 


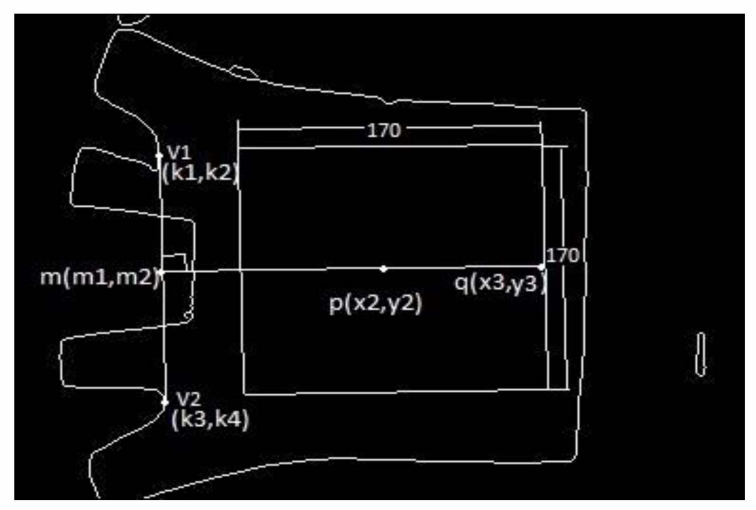

Figure3: ROI Detection

The angle $\alpha$ between the two point's $p$ and $q$ is used to find the orientation of the palm as shown in Figure 4. The image is rotated by angle $\alpha$ in order to correct the wrong orientation. The angle is given by Equation (9)

$\alpha=\tan -1\left(\frac{y 3-y 1}{\mathrm{x} 2-\mathrm{x} 1}\right)$

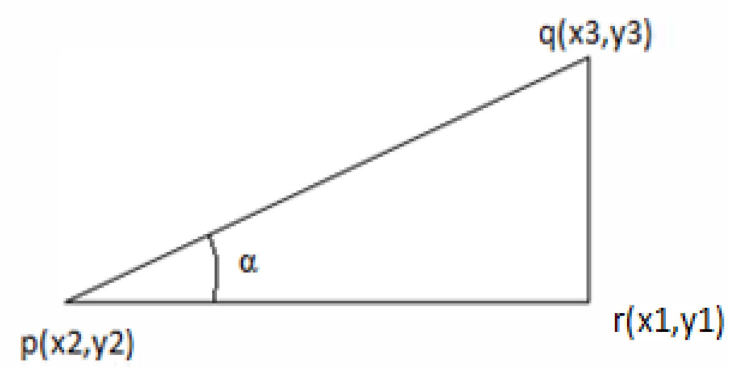

Figure 4: Angle Estimation.

\section{KekreTransform (KT)}

Kekre Transform can be used for easy storage and fast retrieval of images from large databases. The KT matrix can be of any size. The central diagonal values of the matrix and, the upper diagonal values of the matrix are all one and the lower values of the matrix are all zeroes. The KT matrix is shown in figure 5.

$$
\mathrm{K}(\mathrm{x}, \mathrm{y})=\left\{\begin{array}{cc}
1 & , x \leq y \\
-N+(x-1) & , x=y+1 \ldots \\
0 & , x>y+1
\end{array}\right.
$$

\begin{tabular}{|l|l|l|l|l|l|l|l|}
\hline 1 & 1 & 1 & 1 & 1 & 1 & 1 & 1 \\
\hline-7 & 1 & 1 & 1 & 1 & 1 & 1 & 1 \\
\hline 0 & -6 & 1 & 1 & 1 & 1 & 1 & 1 \\
\hline 0 & 0 & -5 & 1 & 1 & 1 & 1 & 1 \\
\hline 0 & 0 & 0 & -4 & 1 & 1 & 1 & 1 \\
\hline 0 & 0 & 0 & 0 & -3 & 1 & 1 & 1 \\
\hline 0 & 0 & 0 & 0 & 0 & -2 & 1 & 1 \\
\hline 0 & 0 & 0 & 0 & 0 & 0 & -1 & 1 \\
\hline
\end{tabular}

Figure 5: 8x8 Kekre Transform matrix

\section{Kekre Wavelet Transform (KWT)}

Kekre Wavelet Transform is obtained from Kekre Transform. Kekre wavelet matrices of dimensions $(2 \mathrm{~N}) X(2 \mathrm{~N}),(3 \mathrm{~N}) X$ $(3 \mathrm{~N}) \ldots\left(\mathrm{N}^{2}\right) \times\left(\mathrm{N}^{2}\right)$ can be constructed from an $\mathrm{N} X \mathrm{~N}$ Kekre Transform matrix. Thus from an $8 \times 8$ Kekre matrix, a 16x16, $24 \times 24 \ldots 64 \times 64$ matrix can be generated. From an $N^{*} \mathrm{~N}$ KT matrix an $\mathrm{M}^{*} \mathrm{M} K W T$ can be generated, so that $\mathrm{M}=\mathrm{N}^{*} \mathrm{P}$ Where $\mathrm{P}$ ranges between 2 and $\mathrm{N}(2 \leq p \leq N)$. We have generated a $64 \times 64 \mathrm{KWT}$ from an $8 \times 8 \mathrm{KT}$ matrix. $\mathrm{M}=64, \mathrm{~N}=8, \mathrm{P}=\mathrm{M} / \mathrm{N}$ $=8$.

\section{- The Temporary Matrix}

Its dimensions are given by the last $(\mathrm{P}-1)$ rows and $\mathrm{P}$ columns of the $8 \times 8$ Kekre Transform matrix which is a $7 \times 8$ matrix as shown in the figure 6 .

\begin{tabular}{|l|l|l|l|l|l|l|l|}
\hline-7 & 1 & 1 & 1 & 1 & 1 & 1 & 1 \\
\hline 0 & -6 & 1 & 1 & 1 & 1 & 1 & 1 \\
\hline 0 & 0 & -5 & 1 & 1 & 1 & 1 & 1 \\
\hline 0 & 0 & 0 & -4 & 1 & 1 & 1 & 1 \\
\hline 0 & 0 & 0 & 0 & -3 & 1 & 1 & 1 \\
\hline 0 & 0 & 0 & 0 & 0 & -2 & 1 & 1 \\
\hline 0 & 0 & 0 & 0 & 0 & 0 & -1 & 1 \\
\hline
\end{tabular}

Figure 6: Temporary Matrix T

\section{- Algorithm used to Obtain KWT Matrix.}

1. Each columns of $\mathrm{KT}$ are repeated $\mathrm{P}=8$ times each generating 64 columns.

2. The first row of temporary matrix is

$[-7,1,1,1,1,1,1,1]$.It is used to generate the next 8 rows. The same digits are repeated in every subsequent 8 columns.

3 . The second row $[0,-6,1,1,1,1,1,1]$ is used to generate the next 8 rows and 64 columns.

4. This process is continued for all rows of temporary matrix, getting a $64 \times 64$ KWT matrix.

The first 16 columns and 18 rows of the $64 \times 64$ Kekre Wavelet Transform Matrix is as seen in Figure 8.

\section{E. Discreet Wavelet Transform (DWT)}

The Hear wavelet which is an orthogonal wavelet is used in our method. It is discontinuous and resembles a step function. It is expressed by the following expressions

$$
\Psi(x)=\left\{\begin{array}{c}
+1 \quad 0 \leq x \leq \frac{1}{2} \\
-1 \quad 1 / 2 \leq x \leq 1 \\
0 \text { otherwise }
\end{array}\right.
$$

Equation $\Phi(\mathrm{x})=\left\{\begin{array}{ccc}1 & 0<=x<=1 \\ 0 & \text { otherwise }\end{array}\right.$ 


\section{F. Features}

\section{- KWT Features}

The feature vector is obtained using the Expression 13.

$[$ KWT $] *[$ Image Matrix $][\text { KWT }]^{\mathrm{T}}$

\section{- DWT Features}

One level Hear wavelet is applied to each row and subsequently to the columns of the resulting image. The features are obtained from LL sub band discarding the other detailed bands.

\section{G. Euclidean Distance:}

The Euclidean Distance is calculated using the equation 14.

$\mathrm{D}(\mathrm{p}, \mathrm{q})=\sqrt{(p i-q i)^{2}+(p j-q j)^{2}}$

Where $\boldsymbol{p} \boldsymbol{i}$ and $\boldsymbol{p} \boldsymbol{j}$ are features of database and $\boldsymbol{q} \boldsymbol{i}$ and $\boldsymbol{q} \boldsymbol{j}$ are features of test image. The Euclidean distance is applied separately on the KWT and DWT features of the test and database images to obtain the match and mismatch counts from which the FAR and FRR are found.

\section{H. EER and TSR}

The EER (Equal Error Rate) and TSR (Total Success Rate) of KWT and DWT are found by plotting the FAR (False Acceptance Rate) and FRR (False Rejection Rate) with respect to different values of threshold.

\section{Fusion}

The EER of the fusion is computed using equation 15 .

FEER $=\left|\frac{\log (E E R 1)}{\log _{2}\left(E_{\left.E R 1^{3}\right)}\right.}-\frac{\log (E E R 2)}{\log _{2}\left(E E R 2^{3}\right)}\right|$

$E E R 1=$ Equal Error Rate for KWT, EER2=Equal Error Rate for DWT

TSR of the fusion is found by the equation 16 .

FTSR $=1-\left|\frac{\log (T S R 1)}{\log _{2}\left(\text { TSR1 }^{3}\right)}-\frac{\log \left(T_{S R 2}\right)}{\log 2\left(\left(\text { TSR2 }^{3}\right)\right.}\right|$

TSR1=Total Success Rate for KWT, TSR2=Total Success Rate for DWT

\section{ALGORITHM}

Problem definition: - Authentication of a person based onpalmprint identification using KWT, DWT and fusing of KWT and DWT score levels at matching stage. The proposed algorithm is given in table 1 .

Table1: Proposed palmprint identification algorithm

Input:palmprint database ,test palmprint
Output: test palmprint identification
1. The Poly U database is considered, and
a database is created to test the
proposed algorithm

2. The normalization, segmentation and histogram equalization are used on palmprint image in preprocessing stage.

3. KWT and DWT are applied on preprocessed image to generate KWT and four subbands of DWT.

4. The KWT and approximation band coefficients of DWT are considered as features.

5. The KWT features of test and database images are compared using ED, to compute EER and TSR.

6. The DWT features of test and database palmprint images are compared using ED to compute EER and TSR.

7. The values of EER obtained from KWT and DWT are fused using log transformation.

$$
\text { FEER }=\left|\frac{\log (E E R 1)}{\log _{2}\left(E E R^{3}\right)}-\frac{\log (\text { EER2 })}{\log _{2}\left(E E R^{3}\right)}\right|
$$

EER1 $=$ Equal Error Rate for KWT

EER2 $=$ Equal Error rate for DWT

8. The values of TSR of KWT and DWT are fused using log transfomation FTSR= $1-\left|\frac{\log (T S R 1)}{\log _{2}\left(T S R^{3}\right)}-\frac{\log (\text { TSR2 })}{\log 2(T S R 2)}\right|$

$\mathrm{TSR} 1=$ total success rate for $\mathrm{KWT}$

TSR2 $=$ total success rate for DWT

\section{PERFORMANCE ANALYSES:}

The Poly U Database is used for performance analysis. The database is created by varying 20 to 100 persons inside database (PID) and persons outside database (POD). The variations of FRR and FAR with threshold values for KWT with PID: POD is 20:100 are plotted in figure 7. With increasing threshold FRR decreases, where as FAR increases. It is observed that, the value of EER is 0.009 at 0.33 threshold value. The variations of FRR and FAR with threshold values for DWT with PID: POD is 20:100 are plotted in figure 8. With increasing threshold FRR decreases, where as FAR increases. It is observed that, the value of EER is 0.1 at 0.46 threshold value.

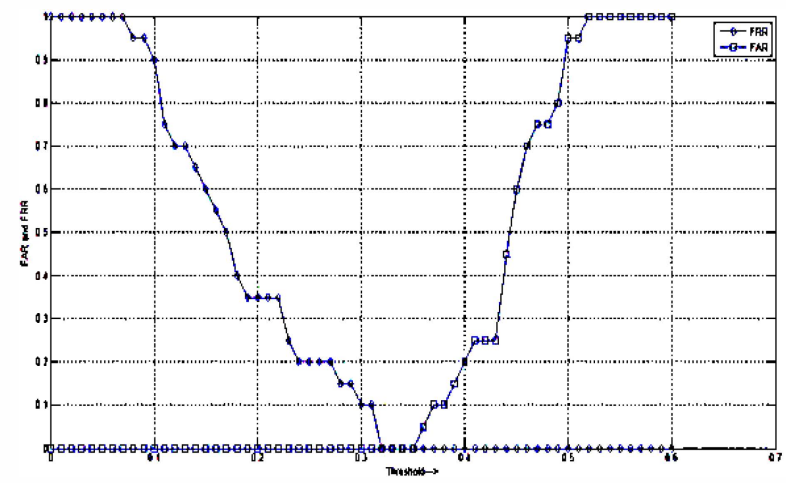

Figure 7: Plot of FAR and FRR V/S threshold for KWT 


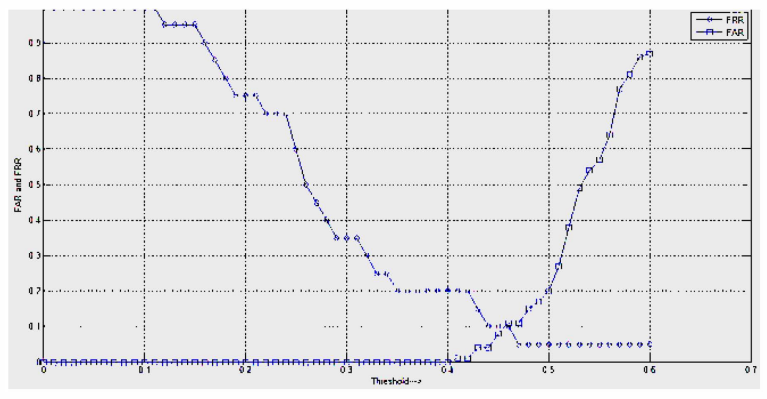

Figure 8: Plot of FAR and FRR V/S threshold for DWT

Table 2: The Variations of EER for KWT, DWT and Proposed Model.

\begin{tabular}{|l|l|l|l|l|}
\hline PID & POD & $\begin{array}{l}\text { EER } \\
\text { (KWT) }\end{array}$ & $\begin{array}{l}\text { EER } \\
\text { (DWT) }\end{array}$ & $\begin{array}{l}\text { EER } \\
\text { (Proposed } \\
\text { Model) }\end{array}$ \\
\hline 20 & 100 & 0.0090 & $\mathbf{0 . 1 0 0}$ & $\mathbf{0 . 0 7 6 9}$ \\
\hline 40 & 80 & $\mathbf{0 . 0 4 2 0}$ & $\mathbf{0 . 1 0 0 0}$ & $\mathbf{0 . 0 4 1 2}$ \\
\hline 60 & 60 & $\mathbf{0 . 0 5 5 0}$ & $\mathbf{0 . 1 1 6 7}$ & $\mathbf{0 . 0 4 1 8}$ \\
\hline 80 & 40 & $\mathbf{0 . 0 7 5 0}$ & $\mathbf{0 . 1 0 0 0}$ & $\mathbf{0 . 0 1 6 7}$ \\
\hline 100 & 20 & $\mathbf{0 . 0 7 5 0}$ & $\mathbf{0 . 1 0 0 0}$ & $\mathbf{0 . 0 1 6 7}$ \\
\hline
\end{tabular}

The variations of EER for KWT, DWT and proposed model are tabulated in Table 2 with variations of PID from 20 to 100 and POD variations from 100 to 20. The values of EER with KWT are lower than DWT. The proposed model has better EER values compared to KWT and DWT.

Table 3: The Variations of TSR for DWT, KWT and Proposed Model.

\begin{tabular}{|l|l|l|l|l|}
\hline PID & POD & $\begin{array}{l}\text { TSR } \\
\text { (KWT) }\end{array}$ & $\begin{array}{l}\text { TSR } \\
\text { (DWT) }\end{array}$ & $\begin{array}{l}\text { TSR } \\
\text { (Proposed } \\
\text { Model) }\end{array}$ \\
\hline 20 & 100 & 99.100 & 90.000 & 0.9984 \\
\hline 40 & 80 & 95.8000 & 90.0000 & 0.9989 \\
\hline 60 & 60 & 94.500 & 88.300 & 0.9989 \\
\hline 80 & 40 & 92.5000 & 90.0000 & 0.9995 \\
\hline 100 & 20 & 92.5000 & 90.0000 & 0.9995 \\
\hline
\end{tabular}

The percentage variations of TSR for KWT, DWT and proposed model are given in Table 3 for different values of PID and POD. It is observed that percentage TSR values are better in case of KWT compared to DWT. The proposed model has better percentage TSR values compared to individual transformations

The percentage TSR values of proposed algorithm are compared with existing algorithms are given in table 4 . The percentage value of TSR is high in the case of proposed algorithm compared to existing algorithms such as Haifeng sang et al., [2], Jingyuguo et al., [3] and Kalifa [4]. The performance of proposed algorithm is better compared to existing algorithms, since the performance parameters are fused using log transformation at the matching stage.

Table4: Comparison of proposed model TSR values with existing algorithms.

\begin{tabular}{|c|l|l|l|}
\hline $\begin{array}{c}\text { Author \& } \\
\text { Reference }\end{array}$ & $\begin{array}{c}\text { Feature } \\
\text { Extraction }\end{array}$ & Matching & $\begin{array}{l}\% \\
\text { TSR }\end{array}$ \\
\hline $\begin{array}{c}\text { Haifeng Sang et } \\
\text { al.[2] }\end{array}$ & $\begin{array}{l}\text { Local Binary } \\
\text { Pattern } \\
\text { (LBP) }\end{array}$ & $\begin{array}{l}\text { Chi- Square } \\
\text { Statistics }\end{array}$ & $\mathbf{9 7 . 0 1 2}$ \\
\hline $\begin{array}{c}\text { Jingyuguo et al } \\
{[3]}\end{array}$ & $\begin{array}{l}\text { Entropy Map } \\
\text { and 2DPCA. }\end{array}$ & $\begin{array}{l}\text { Nearest } \\
\text { neighbor } \\
\text { Classification }\end{array}$ & $\mathbf{7 9 . 6}$ \\
\hline AB khalifa.[4] & $\begin{array}{l}\text { DWT, Gabor } \\
\text { Filter\& Co- } \\
\text { Occurrence } \\
\text { Matrix }\end{array}$ & $\begin{array}{l}\text { SVM. } \\
\text { Best results } \\
\text { by DWT. }\end{array}$ & $\mathbf{9 5 . 2}$ \\
\hline Proposed & $\begin{array}{l}\text { fusion of } \\
\text { KWT and } \\
\text { DWT }\end{array}$ & $\begin{array}{l}\text { Euclidean } \\
\text { Distance }\end{array}$ & $\mathbf{9 9 . 9 5}$ \\
\hline
\end{tabular}

\section{CONCLUSION}

In this paper, Palmprint Identification using Log Transformation of Transform Domain Features is proposed. The palmprint images are preprocessed to generate ROI in palmprint. The features of preprocessed palmprint images are extracted using KWT and DWT. The values of EER and TSR are computed using KWT and DWT techniques. The values of EER and TSR obtained from KWT and DWT are fused using Log transformation to enhance performance. It is observed that the values of EER and TSR are better in the case of proposed algorithm. In future, the DWT can be replaced by Dual Tree complex wavelet Transform.

\section{ACKNOWLEDGEMENT}

We thank the HOD, Principal and management of Dayananda Sagar College of Engineering, and Rayalseema University for their support in accomplishing this work.

\section{REFERENCES}

[1] Dr. Kekre, Archana, Athavale and DipaliSadavarthi,"Algorithm to Generate Kekre's WaveletTransform from Kekre's Transform” ,International Journal of Engineering Science and Technology, Vol.2, PP 756-767, 2010.

[2]Eryun Liu, Anil K Jain, JieTian, "A Course to Fine Minutiae Based Latent Palmprint Matching, "IEEE Transactions on Pattern Analysis and Machine Intelligence, 2013.

[3]Haifeng Sang, Yueshi Ma, Jing Huang, "Robust Palmprint Recognition Based on Touch less Color Palmprint Images Acquired", Scientific Research, journal of Signal and Information Processing, Vol 4 ,PP 134-139, 2013.

[4] Jingyuguo, Yuqin Liu, Weiqi Yuan, Dan Long, "Palmprint Recognition using Entropy Map and2DPCA from a Single image per Person", Journal of Computational Information Systems, PP 3415-3421,2013.

[5]AB Khalifa, "Wavelet,Gabor Filter and Co-occurrence Matrix for Palmprint Verification", International journal of image, graphics and signal processing, Vol 5, PP 1-8, 2013. 